\title{
Systemic inflammation score in locally advanced rectal cancer patients following total mesorectal excision
}

\section{Yanru Feng \\ Luying Liu \\ Yuan Zhu}

Department of Radiation Oncology, Zhejiang Key Laboratory of Radiation Oncology, Zhejiang Cancer Hospital, Hangzhou, People's Republic of China

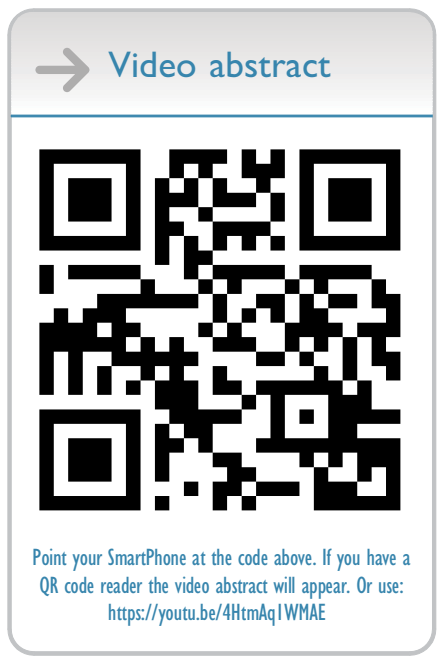

Correspondence: Yuan Zhu Department of Radiation Oncology, Zhejiang Key Laboratory of Radiation Oncology, Zhejiang Cancer Hospital, No. I, East Banshan Road, Gongshu District, Hangzhou 3 10022, People's Republic of China

Tel +86 57188128148

Fax +8657188122062

Email zhuyuan2014I2@I63.com
This article was published in the following Dove Press journal: OncoTargets and Therapy

Objective: The objective of the study was to evaluate whether any association exists between systemic inflammation score (SIS) and adverse events (AEs) and survival of locally advanced rectal cancer patients treated with total mesorectal excision (TME) followed by adjuvant chemoradiotherapy.

Patients and methods: All of the 109 rectal cancer patients recruited between May 2008 and June 2015 were treated with TME followed by adjuvant chemoradiotherapy. The prognostic ability of SIS for overall survival (OS) was calculated by the receiver operating characteristic (ROC) curves.

Results: According to the classification of the SIS, $22(20.2 \%), 59(54.1 \%)$ and $28(25.7 \%)$ patients were classified as a score of 2, 1 and 0 , respectively. With an area under the curve (AUC) of 0.616, the SIS score of 1 was defined as the optimal cut-off value. Therefore, we divided the patients into the SIS-low group (SIS score of 1 or $0, n=87$ ) and SIS-high group (SIS score of 2, $\mathrm{n}=22$ ). Multivariate analysis indicated that SIS was associated with OS (HR $0.390,95 \%$ CI $0.186-0.817, P=0.012$ ). The 5-year OS rate in patients without adjuvant chemotherapy was lower than the patients with adjuvant chemotherapy $(53.3 \%$ vs $75.8 \%$, $P=0.010$ ). Multivariate analysis showed that adjuvant chemotherapy was associated with OS (HR $0.217,95 \%$ CI $0.089-0.529, P=0.001$ ). A marginal statistically significant difference was observed in terms of leukopenia during adjuvant chemoradiotherapy between the SISlow group and the SIS-high group $(P=0.05)$.

Conclusion: These results suggest that SIS might serve as an independent biomarker for predicting AEs and prognosis in locally advanced rectal cancer treated with TME followed by adjuvant chemoradiotherapy. Strengthening treatment may be administered to locally advanced rectal cancer with high SIS score obtained before adjuvant chemoradiotherapy.

Keywords: rectal cancer, SIS, chemoradiotherapy, acute adverse event, survival

\section{Introduction}

Neoadjuvant chemoradiotherapy followed by total mesorectal excision (TME) has been recommended for patients with locally advanced rectal cancer. However, for patients with locally advanced rectal cancer treated by primary surgery, adjuvant chemoradiotherapy is recommended. ${ }^{1}$ Broad variations of survival and adverse events (AEs) have been reported in response to the adjuvant chemoradiotherapy. ${ }^{2}$ In our previous studies, genetic variations in mismatch repair genes and microRNA seed regions could predict the risk of AEs and prognosis. ${ }^{3,4}$

Historically, radiotherapy toxicity was explained using the linear quadratic model. ${ }^{5}$ As the seventh hallmark of cancer, the role of inflammation in radiotherapy 
toxicity needs further study. ${ }^{6}$ The systemic inflammation score (SIS), which is based on lymphocyte-to-monocyte ratio (LMR) and serum albumin level, has been reported to be associated with the prognosis of gastric cancer, renal cell carcinoma and colorectal cancer. ${ }^{7-9}$ Since colon cancer and rectal cancer differ in their biological behaviors, they should be analyzed separately. ${ }^{10,11}$ The aim of this study was to evaluate whether any association exists between SIS and AEs and survival of locally advanced rectal cancer patients treated with TME followed by adjuvant chemoradiotherapy.

\section{Materials and methods}

\section{Patients}

This study was approved by the independent ethics committee, Zhejiang Cancer Hospital. Consent was waived and patient records were deidentified and anonymized prior to analysis. The study was in compliance with the Declaration of Helsinki.

All of the rectal cancer patients recruited between May 2008 and June 2015 were treated with TME followed by a total radiation dose of 45-50.4 Gy in 25-28 fractions at 1.8-2.0 Gy per daily fraction concurrently with capecitabine or capecitabine plus oxaliplatin. The details of adjuvant chemoradiotherapy were described previously. $^{2}$ Patient eligibility criteria included as following: (1) pathological stage II or III rectal adenocarcinoma; (2) R0 TME; (3) Karnofsky Performance Score (KPS) $\geq 70$; (4) 18-75 years old; (5) with no history of cancer excluding carcinoma in situ of the uterine cervix or basal cell carcinoma of the skin; and (6) with adequate organ function. AEs were graded according to the Common Terminology Criteria for Adverse Events ver. 4.0.

\section{Follow-up}

All patients were evaluated weekly during radiotherapy, and were required to be followed-up after the completion of treatment: every 3 months for the first 2 years, and every 6 months thereafter. Each follow-up included physical examination, complete blood count, liver and renal function test and tumor markers (carcinoembryonic antigen and cancer antigen 19-9). Pelvic computed tomography (CT) or magnetic resonance imaging (MRI), chest radiography and abdominal $\mathrm{CT}$ and/or ultrasonography were performed every 6 months. Colonoscopic examination was repeated annually.

\section{Data extraction}

The patient demographic variables (age, sex) and clinical characteristics (KPS, surgical procedure, concurrent oxaliplatin-based chemoradiotherapy, adjuvant chemotherapy, pathological stage, distance from anal verge, results of peripheral blood tests before chemoradiotherapy and related follow-up data) were collected. All patients were staged based on the 8th edition of the American Joint Committee on Cancer staging system. Blood samples were drawn and assayed within 2 weeks before chemoradiotherapy. The laboratory parameters included serum albumin level, lymphocyte and monocyte count. Patients with a serum level $\geq 40 \mathrm{gL}^{-1}$ and $\mathrm{LMR} \geq 4.44$ were classified as a score of 0 ; patients with either a serum albumin level $<40 \mathrm{gL}^{-1}$ or LMR $<4.44$ were classified as a score of 1 ; patients with both hypoalbuminemia $<40 \mathrm{gL}^{-1}$ and LMR $<4.44$ were classified as a score of $2 .^{5-7}$

\section{Statistical analysis}

Statistical analysis was carried out using SPSS version 22.0 (IBM, Armonk, NY, USA). The prognostic ability of SIS for overall survival (OS) was calculated by the receiver operating characteristic (ROC) curves. The disease-free survival (DFS) and OS were measured from the day of surgery to the date of the event and were estimated by use of the Kaplan-Meier method. Log-rank test and Chi-square test were used to assess differences between the SIS-low and the SIS-high group. Multivariate analyses with the Cox proportional hazards model were used to test independent significance by using backward elimination of insignificant explanatory variables. Host factors (age and sex) were included as covariates in all tests. Statistical tests were based on a two-sided significance level. $P<0.05$ indicated statistical significance.

\section{Results}

\section{Characteristics of study subjects}

A total of 109 patients were enrolled; the median follow-up time was 6.6 years (range, 2.4-9.5 years). According to the classification of the SIS, 22 (20.2\%), 59 (54.1\%) and 28 $(25.7 \%)$ patients were classified as a score of 2,1 and 0 , respectively. According to the ROC curve to predict OS, an SIS score of 1 was defined as the optimal cut-off value with an area under the curve (AUC) of 0.616 (Figure 1). Therefore, we divided the patients into the SIS-low group (SIS score of 1 or $0, \mathrm{n}=87$ ) and SIS-high group (SIS score of $2, \mathrm{n}=22$ ). The basic clinical characteristics of the patients are presented in Table 1. 


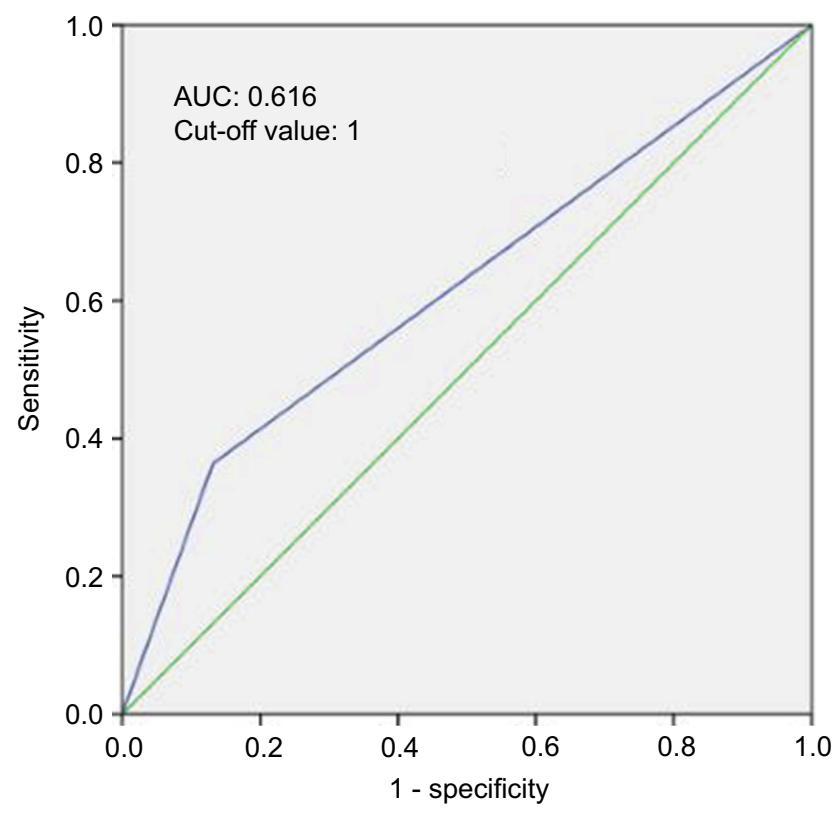

Figure I Receiver operating characteristic curves predicting overall survival by SIS are represented.

Abbreviations: AUC, area under the curve; SIS, systemic inflammation score.

Table I Clinical characteristics

\begin{tabular}{|l|l|l|l|}
\hline Characteristic & $\begin{array}{l}\text { SIS-low } \\
\text { group }\end{array}$ & $\begin{array}{l}\text { SIS-high } \\
\text { group }\end{array}$ & P-value \\
\hline $\begin{array}{l}\text { Sex } \\
\text { Male } \\
\text { Female }\end{array}$ & $\begin{array}{l}56(64.4) \\
31(35.6)\end{array}$ & $\begin{array}{l}12(54.5) \\
10(45.5)\end{array}$ & 0.396 \\
\hline $\begin{array}{l}\text { Age } \\
<54 \\
\geq 54\end{array}$ & $45(51.7)$ & $14(63.6)$ & 0.198 \\
\hline $\begin{array}{l}\text { KPS } \\
90-100\end{array}$ & $42(48.3)$ & $8(36.4)$ & \\
$70-80$ & $59(67.8)$ & $16(72.7)$ & 0.657 \\
\hline $\begin{array}{l}\text { Surgery } \\
\text { Dixon } \\
\text { Mile's }\end{array}$ & $28(32.2)$ & $6(27.3)$ & \\
\hline $\begin{array}{l}\text { Distance from anal verge (cm) } \\
\text { 55 } \\
>5\end{array}$ & $\begin{array}{l}60(69.0) \\
27(31.0)\end{array}$ & $\begin{array}{l}14(63.6) \\
8(36.4)\end{array}$ & 0.632 \\
\hline $\begin{array}{l}\text { Pathological stage } \\
\text { II } \\
\text { III }\end{array}$ & $47(54.0)$ & $13(59.1)$ & \\
\hline $\begin{array}{l}\text { Concurrent oxaliplatin-based } \\
\text { chemoradiotherapy } \\
\text { Yes }\end{array}$ & $\begin{array}{l}13(14.9) \\
\text { No }\end{array}$ & $\begin{array}{l}1(4.5) \\
21(95.1)\end{array}$ & 0.293 \\
\hline
\end{tabular}

(Continued)
Table I (Continued).

\begin{tabular}{|l|l|l|l|}
\hline Characteristic & $\begin{array}{l}\text { SIS-low } \\
\text { group }\end{array}$ & $\begin{array}{l}\text { SIS-high } \\
\text { group }\end{array}$ & P-value \\
\hline $\begin{array}{l}\text { Adjuvant chemotherapy } \\
\text { Yes }\end{array}$ & $15(17.2)$ & $\begin{array}{l}3(13.6) \\
19(86.4)\end{array}$ & $>0.999$ \\
No & $72(82.8)$ & \\
\hline
\end{tabular}

Abbreviations: SIS, systemic inflammation score; KPS, Karnofsky Performance Score.

No statistically significant difference was observed in terms of age, sex, KPS, surgical procedure, concurrent oxaliplatinbased chemoradiotherapy, adjuvant chemotherapy, pathological stage and distance from anal verge between the SIS-low group and the SIS-high group.

\section{SIS and AEs during chemoradiotherapy}

The most frequently observed acute toxicity during chemoradiotherapy was mainly grade 0 or grade 1 . The incidence of acute grade $\geq 2$ diarrhea, dermatitis and leukopenia was $36.7 \%, 18.3 \%$ and $24.8 \%$, respectively. A marginal statistically significant difference was observed in terms of leukopenia during chemoradiotherapy between the SIS-low group and the SIS-high group $(P=0.05)$ (Table 2$)$.

\section{SIS and survival}

The 5-year OS rate in the SIS-high group was lower than the patients in the SIS-low group $(50.0 \%$ vs $78.2 \%$, $P=0.011$ ). No statistically significant difference was found in terms of 5-year DFS rate between the SIS-low and the SIS-high groups $(66.0 \%$ vs $52.5 \%, P=0.281)$ (Figure 2). Multivariate analysis by Cox proportionalhazards model showed that SIS was associated with OS (HR 0.337, 95\% CI 0.160-0.713, $P=0.004$ ) (Table 3). The 5 -year OS rate in patients without adjuvant chemotherapy was lower than the patients with adjuvant chemotherapy (53.3\% vs $75.8 \%, P=0.010$ ). Multivariate analysis showed that adjuvant chemotherapy was associated with OS (HR $0.217,95 \%$ CI $0.089-0.529, P=0.001)$.

\section{Discussion}

In the present study, we evaluated the association between systematic inflammation and AEs and survival of locally advanced rectal cancer patients treated with TME followed by adjuvant chemoradiotherapy. To our knowledge, the present study is the first study to evaluate the role of SIS for AEs and survival in locally advanced rectal cancer patients treated with TME followed by adjuvant chemoradiotherapy. The 
Table 2 Association between SIS and acute adverse events in patients with rectal cancer receiving chemoradiation therapy

\begin{tabular}{|c|l|l|l|}
\hline Characteristic & SIS-low group & SIS-high group & $P$-value \\
\hline $\begin{array}{c}\text { Leukopenia } \\
\text { Grade } \geq 2 \\
\text { Grade }<2\end{array}$ & $\begin{array}{l}18(20.7) \\
69(79.3)\end{array}$ & $\begin{array}{l}9(40.9) \\
13(59.1)\end{array}$ & 0.050 \\
\hline $\begin{array}{c}\text { Dermatitis } \\
\text { Grade } \geq 2\end{array}$ & $15(17.2)$ & $5(22.7)$ & 0.553 \\
Grade $<2$ & $72(82.8)$ & $17(77.3)$ & \\
\hline $\begin{array}{c}\text { Diarrhea } \\
\text { Grade } \geq 2\end{array}$ & $33(37.9)$ & $7(31.8)$ & 0.595 \\
Grade $<2$ & $54(62.1)$ & $15(68.2)$ & \\
\hline
\end{tabular}

Abbreviation: SIS, systemic inflammation score.

results suggested that SIS was an independent prognostic predictor of OS and marginal statistically significant difference was observed in terms of leukopenia between the SISlow group and the SIS-high group during chemoradiotherapy.

As Virchow originally made links between inflammation and cancer in 1863, the role of systemic inflammation in the pathogenesis and progression of cancer has been revived in the recent years. ${ }^{12,13}$ Lymphocyte, a surrogate marker of host immunological status, has been reported to suppress tumor progression and promote tumor immune-surveillance. ${ }^{14-17}$ Tumor-associated macrophages, derived from monocytes, have been reported to play a critical role in the pathogenesis, progression and therapeutic resistance of cancer. ${ }^{18,19}$ LMR was reported to be associated with the prognosis of rectal cancer with or without neoadjuvant chemoradiotherapy. ${ }^{20-23}$ Serum albumin level is an indicator of nutritional status and systematic inflammation response, ${ }^{24}$ associated with prognosis and postoperative morbidity in rectal cancer. ${ }^{25-27}$

In the study including 441 clear-cell renal cell carcinoma patients undergoing nephrectomy, SIS was firstly reported to be associated with tumor behaviors and OS after adjusting for traditional clinicopathological features and other markers of systemic inflammation responses. ${ }^{7}$ The higher SIS score was also associated with poorer OS and DFS in gastric cancer patients after curative gastrectomy. ${ }^{9}$ In the study of evaluating the prognostic impact of the SIS in colorectal cancer patients after curative resection, 161 (22.1\%) patients with rectal cancer who did not receive neoadjuvant chemoradiotherapy or radiation therapy were included. SIS was significantly associated with more advanced stage. Furthermore, SIS was an independent prognostic factor for OS by multivariate analysis. Compared with SIS $=0$, the hazard ratio of SIS $=1$ was 1.711 $(P=0.030)$, and that of SIS $=2$ was $1.944(P=0.018) .{ }^{8}$ In our study, the prognostic ability of SIS for OS was calculated by the ROC curves and we divided the patients into the SISlow group (SIS score of 1 or $0, \mathrm{n}=87$ ) and SIS-high group (SIS score of 2, $\mathrm{n}=22$ ) for the limited patients. No statistically significant difference was observed in terms of pathological stage between the SIS-low group and the SIS-high group; however, more patients with Stage III were classified into the SIS-high group ( $95.5 \%$ vs $85.1 \%, P=0.293$ ). Multivariate analysis showed that SIS was an independent prognostic predictor of OS, which was similar with the previous report of colorectal cancer.

The mechanisms of radiotherapy toxicity include direct or indirect cell death, inflammation and immune reaction. Genetic analyses and radiomics had been adopted for predicting radiotherapy toxicity. ${ }^{6}$ In our previous studies, ${ }^{3,4}$ MSH3 rs 12513549 (odds ratio, 0.68; 95\% CI, 0.48-0.97; $P=0.037$ ), rs33013 (odds ratio, 0.71; 95\% CI, 0.50-0.99; $p=0.049$ ) and rs6151627 (odds ratio, $1.51 ; 95 \%$ CI, 1.02 $2.27 ; P=0.041)$ were significantly associated with grade $\geq 2$ diarrhea; PMS1 rs1233255 A>C (odds ratio, 0.48; 95\% CI,

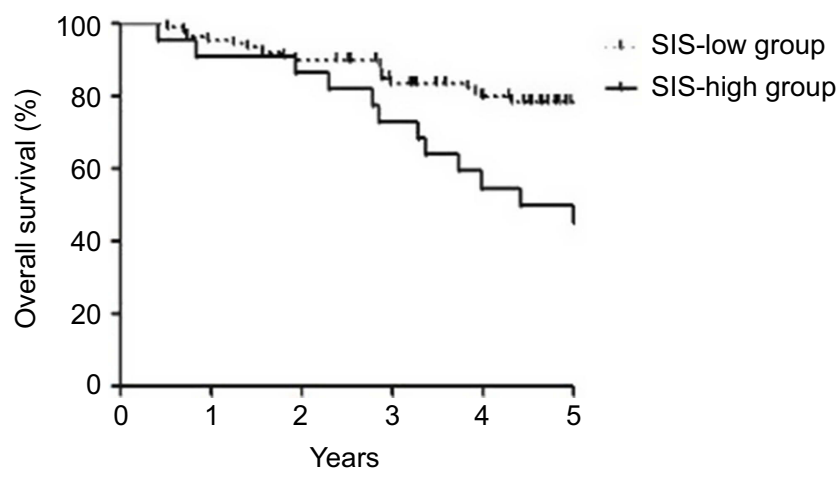

Figure 2 Kaplan-Meier curves of overall survival and disease-free survival in the SIS-low group and the SIS-high group. Abbreviation: SIS, systemic inflammation score. 
Table 3 Multivariate analysis of overall survival by Cox proportional hazards model

\begin{tabular}{|l|l|l|l|}
\hline \multirow{2}{*}{ Items } & \multicolumn{2}{l|}{ Overall survival } & P5\% Cl \\
\cline { 2 - 4 } & HR & $0.160-0.713$ & $0.004^{*}$ \\
\hline SIS (SIS-low group vs SIS-high group) & 0.337 & $0.021-1.212$ & 0.076 \\
Pathological stage (II vs III) & 0.160 & $1.226-5.671$ & $0.013^{*}$ \\
KPS (70-80 vs 90-100) & 2.637 & $0.089-0.529$ & $0.00 I^{*}$ \\
Adjuvant chemotherapy (Yes vs No) & 0.217 & \\
\hline
\end{tabular}

Note: $* P<0.05$.

Abbreviations: SIS, systemic inflammation score; KPS, Karnofsky Performance Score; Cl, confidence interval; HR, hazard ratio.

$0.23-0.93 ; P=0.041)$ was associated with grade $\geq 2$ dermatitis; single nucleotide polymorphism rs2273626 was associated with grade $\geq 2$ leukopenia (odds ratio, $0.48 ; 95 \% \mathrm{CI}$, $0.31-0.74 ; P=0.0009)$. In the present study, a marginal statistically significant difference was observed in terms of grade $\geq 2$ leukopenia during radiotherapy between the SIS-low group and the SIS-high group.

The serum albumin level and white blood cell count were obtained before surgery in the previous studies of SIS. $^{7-9}$ In the present study, the serum albumin level and white blood cell count obtained before adjuvant chemoradiotherapy were used to evaluate the role of SIS for AEs and survival in locally advanced rectal cancer patients treated with TME followed by adjuvant chemoradiotherapy. In the study including 146 rectal cancer patients treated by neoadjuvant chemoradiotherapy and surgery, the cutpoint was significantly associated with OS for LMR before neoadjuvant chemoradiotherapy but not for LMR after neoadjuvant chemoradiotherapy. ${ }^{22}$ The role of SIS before any treatment in rectal cancer should be elucidated in the future.

There were several limitations to our study. First, for the limited patients from one institution, the role of the SIS in rectal cancer should be evaluated prospectively in other populations and larger cohorts in the future. Second, although circulating white blood cell counts are thought to be an indicator of systematic immune reaction, we did not determine the relation of SIS and other immunological characteristics, such as immunoscore, ${ }^{28}$ which has been reported to be a prognostic marker of rectal cancer. Third, despite no head-to-head comparison in the adjuvant setting, current treatment guidelines of rectal cancer recommend either XELOX or FOLFOX as standard of care treatment options. ${ }^{1}$ Multivariate analysis showed that adjuvant chemotherapy was associated with OS in the present study. In the SIS-high group, only 3 patients received adjuvant chemotherapy; it would be inappropriate to evaluate the role of adjuvant chemotherapy for locally advanced rectal cancer with high SIS score.

\section{Conclusion}

These results suggest that SIS might serve as an independent biomarker for predicting AEs and prognosis in locally advanced rectal cancer treated with TME followed by adjuvant chemoradiotherapy. Strengthening treatment may be administered to locally advanced rectal cancer with high SIS score obtained before adjuvant chemoradiotherapy.

\section{Acknowledgment}

This work was supported by a grant from the Natural Science Foundation of Zhejiang Province (No. LQ19H160003).

\section{Disclosure}

The authors report no conflicts of interest in this work.

\section{References}

1. Benson AB 3rd, Venook AP, AI-Hawary MM, et al. NCCN guidelines version 1. 2019. Available from: http://www.nccn.org/. Accessed July 31, 2019.

2. Feng YR, Zhu Y, Liu LY, et al. Interim analysis of postoperative chemoradiotherapy with capecitabine and oxaliplatin versus capecitabine alone for pathological stage II and III rectal cancer: a randomized multicenter phase III trial. Oncotarget. 2016;7(18):25576-25584. doi:10.18632/oncotarget.8226

3. Huang Y, Feng Y, Ren H, et al. Associations of genetic variations in microRNA seed regions with acute adverse events and survival in patients with rectal cancer receiving postoperative chemoradiation therapy. Int J Radiat Oncol Biol Phys. 2018;100(4):1026-1033. doi:10.1016/j.ijrobp.2017.12.256

4. Yang J, Huang Y, Feng Y, et al. Associations of genetic variations in mismatch repair genes MSH3 and PMS1 with acute adverse events and survival in patients with rectal cancer receiving postoperative chemoradiotherapy. Cancer Res Treat. 2018. doi:10.4143/crt.2018. 527

5. Fowler JF. The linear-quadratic formula and progress in fractionated radiotherapy. Br J Radiol. 1989;62(740):679-694. doi:10.1259/00071285-62-740-679

6. De Ruysscher D, Niedermann G, Burnet NG, et al. Radiotherapy toxicity. Nat Rev Dis Primers. 2019;5(1):13. doi:10.1038/s41572019-0064-5 
7. Chang Y, An H, Xu L, et al. Systemic inflammation score predicts postoperative prognosis of patients with clear-cell renal cell carcinoma. Br J Cancer. 2015;113(4):626-633. doi:10.1038/bjc.2015.241

8. Suzuki Y, Okabayashi K, Hasegawa H, et al. Comparison of preoperative inflammation-based prognostic scores in patients with colorectal cancer. Ann Surg. 2018;267(3):527-531. doi:10.1097/ SLA.0000000000002115

9. Ma M, Weng M, Chen F, et al. Systemic inflammation score is a prognostic marker after curative resection in gastric cancer. ANZ J Surg. 2019;89(4):377-382. doi:10.1111/ans.15103

10. Wei EK, Giovannucci E, Wu K, et al. Comparison of risk factors for colon and rectal cancer. Int $J$ Cancer. 2004;108(3):433-442. doi:10.1002/ijc.11540

11. Nazha B, Moussaly E, Zaarour M, Weerasinghe C, Azab B. Hypoalbuminemia in colorectal cancer prognosis: nutritional marker or inflammatory surrogate? World J Gastrointest Surg. 2015;7 (12):370-377. doi:10.4240/wjgs.v7.i12.370

12. Balkwill F, Mantovani A. Inflammation and cancer: back to Virchow? Lancet. 2001;357:539-545. doi:10.1016/S0140-6736(00)04046-0

13. McMillan DC. The systemic inflammation-based Glasgow Prognostic Score: a decade of experience in patients with cancer. Cancer Treat Rev. 2013;39:534-540. doi:10.1016/j.ctrv.2012.08.003

14. Dunn GP, Old LJ, Schreiber RD. The immunobiology of cancer immunosurveillance and immunoediting. Immunity. 2004;21:137148. doi:10.1016/j.immuni.2004.07.017

15. Migita K, Takayama T, Saeki K, et al. The prognostic nutritional index predicts long-term outcomes of gastric cancer patients independent of tumor stage. Ann Surg Oncol. 2013;20(8):2647-2654. doi: $10.1245 / \mathrm{s} 10434-013-2926-5$

16. Eo WK, Jeong DW, Chang HJ, et al. Absolute monocyte and lymphocyte count prognostic score for patients with gastric cancer. World $J$ Gastroenterol. 2015;21(9):2668-2676. doi:10.3748/wjg.v21.i9.2668

17. Johnson ME, Zhu F, Li T, et al. Absolute lymphocyte count: a potential prognostic factor for Merkel cell carcinoma. J Am Acad Dermatol. 2014;70(6):1028-1035. doi:10.1016/j.jaad.2014.01.890

18. Aras S, Zaidi MR. TAMeless traitors: macrophages in cancer progression and metastasis. Br J Cancer. 2017;117:1583-1591. doi:10.1038/bjc.2017.356
19. Ruffell B, Coussens LM. Macrophages and therapeutic resistance in cancer. Cancer Cell. 2015;27:462-472. doi:10.1016/j.ccell.2015. 02.015

20. Deng YX, Lin JZ, Peng JH, et al. Lymphocyte-to-monocyte ratio before chemoradiotherapy represents a prognostic predictor for locally advanced rectal cancer. Onco Targets Ther. 2017;10:55755583. doi:10.2147/OTT.S146697

21. Abe S, Kawai K, Nozawa H, et al. LMR predicts outcome in patients after preoperative chemoradiotherapy for stage II-III rectal cancer. $J$ Surg Res. 2018;222:122-131. doi:10.1016/j.jss.2017.09.053

22. Ward WH, Goel N, Ruth KJ, et al. Predictive value of leukocyte- and platelet-derived ratios in rectal adenocarcinoma. J Surg Res. 2018;232:275-282. doi:10.1016/j.jss.2018.06.060

23. Xiao WW, Zhang LN, You KY, et al. A low lymphocyte-to-monocyte ratio predicts unfavorable prognosis in pathological T3N0 rectal cancer patients following total mesorectal excision. $J$ Cancer. 2015;6(7):616-622. doi:10.7150/jca.11727

24. McMillan DC. Systemic inflammation, nutritional status and survival in patients with cancer. Curr Opin Clin Nutr Metab Care. 2009;12 (3):223-226. doi:10.1097/MCO.0b013e32832a7902

25. Hardt J, Pilz L, Magdeburg J, et al. Preoperative hypoalbuminemia is an independent risk factor for increased high-grade morbidity after elective rectal cancer resection. Int J Colorectal Dis. 2017;32 (10):1439-1446. doi:10.1007/s00384-017-2884-7

26. Lohsiriwat V, Lohsiriwat D, Boonnuch W, et al. Pre-operative hypoalbuminemia is a major risk factor for postoperative complications following rectal cancer surgery. World J Gastroenterol. 2008;14 (8):1248-1251. doi:10.3748/wjg.14.1248

27. Chandrasinghe PC, Ediriweera DS, Kumarage SK, et al. Pre-operative hypoalbuminaemia predicts poor overall survival in rectal cancer: a retrospective cohort analysis. BMC Clin Pathol. 2013;13:12. doi:10.1186/1472-6890-13-12

28. Anitei MG, Zeitoun G, Mlecnik B, et al. Prognostic and predictive values of the immunoscore in patients with rectal cancer. Clin Cancer Res. 2014;20(7):1891-1899. doi:10.1158/1078-0432.CCR-13-2830

\section{Publish your work in this journal}

OncoTargets and Therapy is an international, peer-reviewed, open access journal focusing on the pathological basis of all cancers, potential targets for therapy and treatment protocols employed to improve the management of cancer patients. The journal also focuses on the impact of management programs and new therapeutic agents and protocols on patient perspectives such as quality of life, adherence and satisfaction. The manuscript management system is completely online and includes a very quick and fair peer-review system, which is all easy to use. Visit http://www.dovepress.com/ testimonials.php to read real quotes from published authors. 